Poincare Journal of Analysis E Applications

Vol. 2014 (2), 55 - 61

(C) Poincare Publishers

\title{
NORMAL VE RECTIFYING CURVES IN THE EQUIFORM DIFFERENTIAL GEOMETRY OF $G_{3}$
}

\author{
SEZAI KIZILTUĞ, SEMRA YURTTANÇIKMAZ ${ }^{\dagger}$ AND ALI ÇAKMAK
}

$\begin{array}{lll}\text { Date of Receiving } & : & 30.06 .2014 \\ \text { Date of Revision } & : & 12.08 .2014 \\ \text { Date of Acceptance } & : & 04.09 .2014\end{array}$

\begin{abstract}
In this study, we define normal and rectifying curves in the equiform differential geometry of the Galilean space. Furthermore, we obtain some characterizations for normal and rectifying curves in the equiform geometry of the Galilean space G.
\end{abstract}

\section{Introduction}

A Galilean space may be considered as the limit case of a pseudo-Euclidean space in which the isotropic cone degenerates to a plane. This limit transition corresponds to the limit transition from the special theory of relativity to classical mechanics. On the other hand, Galilean space-time plays an important role in nonrelativistic physics. The fact that the fundamental concepts such as velocity, momentum, kinetic energy, etc. and principles; laws of motion and conservation laws of classical physics are expressed in terms of Galilean space[4]. As it is well known geometry of space is associated with mathematical group. The idea of invariance of geometry under transformation group may imply that, on some spacetimes of maximum symmetry there should be a principle of relativity, which requires the invariance of physical laws without gravity under transformations among inertial systems. Besides, theory of curves and the curves of constant curvature in the equiform differential geometry of the isotropic spaces $I_{3}^{1}$ and $I_{3}^{2}$, and the Galilean space $G_{3}$ are described in [1] and [2], respectively. Although the equiform geometry has minor importance related to usual one, the curves that appear here in the equiform geometry, can be seen as generalizations of well-known curves from above mentioned geometries and therefore could have been of research interest.

The purpose of the present paper is to find necessary conditions for normal and rectifying curves in the equiform geometry of the Galilean space $G_{3}$.

2010 Mathematics Subject Classification. 53A04, 53A35.

Key words and phrases. Normal curve, Rectifying curve, Equiform differential geomertry, Galilean space.

Communicated by. S.K. Kaushik

${ }^{\dagger}$ Corresponding author. 